Supernova Shells and Their Birth Events (Lecture Notes in Physics, Vol 316)

(Proceedings of a Workshop held at Physikzentrum Bad Honnef, March 7-11, 1988) edited by Wolfgang Kundt

Springer-Verlag: Berlin-Heidelberg-New York-London-Paris-Tokyo, 1989

viii +253 pages ; price : DM 55 (Hard cover) ; ISBN 3-540-50435-4

Movies made of the expanding fireballs of nuclear explosions were the haunting images of the cold war era. Astronomers are now attempting to make movies of exploding stars in the sky that are many orders of magnitude more powerful than the man made explosions. Radio telescopes strung together in Very Long Baseline Interferometry (VLBI) are being used for such purposes. These and an assortment of other topics concerning supernova explosions and supernova remnants appear in the book which is a Proceedings of a Workshop held at Bad Honnef, Germany in March 1988. The articles in this book are grouped in four sections which deal with (1) general properties of supernova shells and their interpretations ; (2) a few special objects studied in radio, infra-red, optical and X-ray bands; (3) supernova explosion mechanisms and the nature of supernova progenitors ; and (4) on topics concerning the interstellar medium and cosmic ray spectra produced by supernova remnants. A notable feature about this book is that a substantial portion of it is devoted to opposing viewpoints, including minority views, on a number of topics. In the words of the editor, "it is not always easy to find out about them because minority views tend to be ignored in the reviews and prefaces tend to leave the reader with the impression that the reviews express the new scientific consensus".

Particularly interesting among this category is the pair of articles by Sidney van den Bergh and Nino Panagia on the nature of the progenitors of type lb supernovae. The former argues that the spectra, frequency and spatial distribution of supernovae are all consistent with the hypothesis that SNIb have progenitor masses $M>15 M_{O}$ and that type II SNe are produced by stars that have main sequence masses in the range $8 M_{\circlearrowleft}<M<15 M_{O}$ whereas the latter argues that SNIb progenitors are likely to be moderately massive stars $\left(M \sim 7 M_{(}\right)$in binaries. An important link in the argument of van den Bergh is the association of SNIb explosions will HII regions. The highest intrinsic frequency of SNIb and SNII also happen to be in spiral galaxies of type Sc suggesting that both have massive progenitors. On the other hand, Panagia challenges the very association of $\mathrm{Ib}$ with $\mathrm{HII}$ regions both on statistical grounds by comparing the corresponding association of SNIIs with HII regions, as well as by arguing that the association is in some cases only close proximity. However, only giant HII regions can be observed in distant spirals and quite a few SNIb might still be located in intrinsically faint HII regions. Deep searches for HII regions in spirals would be important input to this debate. 
In the same vein, Rino Bandiera challenges the standard picture of Kepler's supernova as having originated from a Population II star and argues that Kepler's progenitor was a runaway star with a high original mass most of which was lost in a wind-a prime example of which is a Wolf-Rayet (WR) star in a binary. Runaway WR stars are typically single-line spectroscopic binaries, having an unseen companion (e.g. a neutron star) orbiting with a period of a few days. If Kepler's SN was actually due to the explosion of the WR star it must have appeared as a type Ib SN. This was preceded by a phase of a massive $X$-ray binary and there is evidence from study of Kepler's supernova remnant (SNR) that the star was subject to a typical red supergiant wind for some time, perhaps in the Roche-lobe filling stage of the massive X-ray binary. All in all, this is the most well developed article in the book among the class representing non-standard views and the author goes through a detailed analysis of the origin and nature of the optical knots, the nature of the dense medium in which the supernova shock expands, the distance to the remnant and the peak absolute magnitude of the $\mathrm{SN}$ which he argues to be more than a magnitude fainter than a normal type ISN (i.e. type la).

Wolfgang Brinkman reviews the $\mathrm{X}$-ray observations of supernova remnants and discusses the problems of deducing chemical abundances in SNRs. Notable among these are the uncertain knowledge of the underlying $X$-ray continuum which affects the equivalent widths of the individual lines, and uncertain reaction rates for many elements that are recuired in non-equilibrium ionisation calculations coupled with the hydrodynamics of shock expanding into the circumstellar medium. Previous calculations based upon equilibrium models of plasma and of local ionisation equilibrium have led to an overabundance of oxygen burning elements and an underabundance of iron, as well as too high X-ray emitting masses for SNRs observed by the Einstein Observatory. However, the practical problems of computing non-equilibrium ionisation evolution of SNRs ars manifold. They range from excessive computing time required to evolve the SNR past the relatively early stage to the physical uncertainities in the underlying initial models of type 1 explosions, e.g. the velocity field of the deflagration front. Furthermore, onedimensional hydrocodes are inadequate to estimate the degree of element mixing in the envelope of the exploding star-these require 2-D and 3-D codes. For example, unless there is a substantial mixing in the initial model, the Fe-line region in the observed spectrum of Tycho's SNR cannot be fitted properly and the equivalent width of $\mathrm{Fe}$ lines are far too narrow despite the fact that the same initial model contains some $0.6 \mathrm{M}_{\odot}$ of iron. On the observational front, radially resolved high resolution spectra in a number of SNR (so far unavailable for most SNRs) may help in pinning down the physical parameters of the SNR accurately.

The first article of Wolfgang Kundt deals with the interpretation of supernova shells and comments on velocity fields, ages, sizes and fine structure of SN shells. It contains a valuable collection of reproduced figures that give the proper motions of quasi-stationary flocculi, radio features and optical knots in Cas $A$ as well as of 
$H_{\alpha}$ bright spots in the Crab nebula. Kundt points out the observations that are believed to be against the standard picture of Supernova shells described by "blast waves" or Sedov-Taylor shock waves which sweep up their circumstellar material. He argues instead that SN explosions resemble "splinter bombs" vvith low filling factor and very little turbulence rather than the "pressure bombs" characteristic of the blast-wave description. This article also contains an interesting aside about the question of the decay of neutron star magnetic fields-an area whose "standard picture" has undergone substantial changes during recent times. Kundt's second article deals with supernova dynamics and light curves and argues that a magnetic torque transfers the angular momentum of the collapsing core and subsequently converts into relativistic pair plasma which drives the supernova piston. Unfortunately the first part of this article is not very well developed beyond some dimensional estimates and lacks the analytical framework of the core bounce and shock driven explosion mechanism (presented by Jim Lattimer in this volume). Although the latter class of models has well-known difficulties, perhaps the last word on spherically symmetric core bounce and resultant hydrodynamic shock driven explosion mechanism (assisted by neutrinos or otherwise) isn't still in and it is premature to conclude that they necessarily fail.

The book also contains a number of papers giving observational result on SNRs. A valuable example is the article by Douglas Milne and others describing high resolution radio observations of SNRs G316.3-0.0 and G332.4+0.1. Here, maps of electric polarization vectors, projected magnetic fields and rotation measures are presented for both of these SNRs. The authors argue that the remnant expanding spherically blows out into a low density cavity-most likely a wind driven bubble from the presupernova stage-and draws in the magnetic field along with it.

On the question of gravitational collapse of a massive star's core and explosion and the associated burst of neutrinos, calculations from hydrodynamical models are compared with the characteristics of neutrino events recorded from SN 1987A in the article by Jim Lattimer. The basic agreement of the neutrino flux and spectrum and the recorded arrival times with those of cooling neutron stars strongly suggest that gravitational collapse resulted in the formation of a hot neutron star and neutrinos could only diffuse out of a dense object over a period of several seconds. The article systematically describes many of the ingredients of the "standard" model calculations of supernova explosions-mediated by hydrodynamic shocks. However in view of the lack of clear success in producing explosions in computer simulations for a wide range of progenitor masses, many unanswered questions remain. For example, is the shock produced in the core bounce powerful enough to blow off the star's envelope in a timescale of few tens of milliseconds with or without the assistance of neutrinos, or do the neutrinos provide a secondary energy source that will effectively re-energize a stalled shock after a "pause (of $=500 \mathrm{~ms}$ ) that refreshes" ? The detection of neutrinos from SN 1987A by the IMB and Kamiokande II detectors was fortunate in that both these experiments 11 
had undergone crucial upgrades only about a year before the neutrinos arrived. Nevertheless, the number of events detected by both these experiments were too small to conclusively settle such questions regarding explosion mechanisms. A supernova explosion in the galactic centre (8.5 kiloparsecs away) will produce an almost hundred-fold increase in integrated signal, depending on detector threshold, sensitivity etc. The Sudbury Neutrino Observatory for example, might detect some 1100 neutrinos in a burst from a SN in the galactic center. Although the last ohservable supernova in our galaxy was in the year 1604 AD (Kepler's SN), it is likely that $\mathrm{SNe}$ actually go off more frequently than once in $\sim 300$ years. Any such events occurring near the galactic center would be visually obscured but would remain detectable in the neutrino channel. The estimate of the rate of SN events in the galaxy is to some extent uncertain. But from the statistical propert es of supernova remnants recently identified from a deep radio continuum survey with the Effelsberg 100 metre telescope, Wolfgang Reich and Ernst Furst conclude in this book that the birthrate of the shell-type remnants is one in 4 to 19 years in our galaxy. A neutron star-producing explosion in our galaxy might therefore require a wait of the order of 10 years. Supernova model builders think that the wait to detect those galactic neutrinos will be well worth it.

$A R_{A Y}$

Tato Institute of Fundomental Research, Homi Bhabho Road, Bomboy-400 005

\section{Quantum Theory and Pictures of Reality: Foundations, Interpretations and New Aspects \\ edited by W Schommers \\ Springer-Verlag: Berlin-Heidelberg-New York-London-Paris-Tokyo-Hong Kong, 1989 \\ xviii + 336 pages, 31 figures ; price : DM 98 (Soft cover) ; ISBN 3-540-50152-5}

"Quantum mechanics - that mysterious, confusing discipline, which none of us really understands but which we know how to use". In a lecture in 1981 $M$ Gellman expressed his feelings about quantum mechanics in the above words. Previous to quantum mechanics, any major theoretical development in physics served two purposes. It gave the rules from which results of experiments could be calculated. Also, it gave a description or a picture of the objective world as it ' evolves undisturbed by any observation. Quantum mechanics seems to have furnished only the first part. This perhaps is at the root of the unhapiness about quantum mechanics, which is shared by most physicists.

Looked at as mechanics of $\psi$-function quantum mechanics is simple and straight for Nard. Given an initial function its time development is uniquely determined by Sshrödinger's equation. In this sense, it is as deterministic as 
classical mechanics. But $\psi$-function can not be regarded as a basic element of observation. What we observe in experiments are not $\psi$-functions but properties of particles or wave fields. In classical mechanics basic elements of observation (such as position momentum etc) are also the basic elements of the theoretical description. In quantum mechanics however the basic elements of the theory (the $\psi$-function) is connected to the basic elements of observation by rules which because of their mysteriousness make it almost impossible to develop any description of the physical reality in terms of observed entities.

The book under review is concerned with the foundation and interpretation as well as with the ne'N aspects of quantum theory. The editor, at the end of the first article on Evolution of Quantum Theory writes "Although the complete formalism of non-relativistic quantum theory was constructed in 1925-26 the interpretation of quantum theory to-day even 60 years after the formulation by far the most controversial problem in current research in the foundations of physics. There is obviously an immense diversity of opinion".

The book consists of six chapters written by four authors. These are (1) Evolution of Quantum Theory by W Schommers (2) The EPR Paradox Roots and Ramifications by P H Eberhard, (3) Non-separability and the Tentative Description of Reality by B d'Espagnat. (4) A Realistic Model for Quantum Theory with a Locality Property by P H Eberhard, (5) Space Time and Quantum Phenomena by W Schommers and (6) Wave-Particle Duality: Recent Proposals for the Detection of Empty Waves by F Selleri.

The first three chapters (Part I of the book) deal with the foundations and interpretations of the usual quantum theory. The last three chapters (Part II of the book) deal with the new aspects and provide information on quantum 'weirdness'. They reflect more or less the individual author's own activities.

In Chapter 1, Schommers gives a brief review of the development of ideas in quantum theory. It is well written and its value is enhanced by occasional critical comments. Commenting on objectivity in quantum theory the authors writes "the human intention influences the structure of reality". The positions of an electron as revealed by an experiment at an instant does not imply that the electron actually occupied that definite position at that instant, prior to the experiment. If instead one chose to measure the momentum, it would seem the electron had a definite momentum and not position at that instant. It seems a better way to present the dilemma would be to assert that in quantum theory all elements of observation cease to be part of the objective reality. As we know, the notion of what constitute objective reality is itself evolving with physics. In quantum mechanics only the statistical averages (not individual observations) constitute what we may call the reproducible objective reality.

In Chapters 2 and 3 the authors concentrate on the more philosophically oriented question of interpretation of quantum theory. The focus is naturally on 
EPR paradox and Bell's inequality, Bell constructed his inequality on the assumption that some basic a priori notions about reality put forward in the EPR paper (impossibility of faster than light effects, locality etc) are correct. Experimental disproof of Bell's inequality therefore implies that all of these notions can not be correct. Eberhard discusses the possibilities which are still open. d'Espagnat in Chapter 3 discusses more or less the same questions but he inducts concepts from modal logic and makes as a result the discussion is more abstract. His final conclusion is that if one accepts-hidden variables do not exist (i.e. it is impossible to produce dispersion free states) then one is almost forced to the notion that quantum states do not have any reality apart from that of being useful predictive tools. The surviving world views are then unavoidably very strange and partake from metaphysics.

In the last three chapters attempts are made to build models of theories which allow some picture about reality while keeping quantum results in tact. For example in Chapter 4 a model is discussed which implies faster than light effects but not instantaneous. By adjusting the parameters in the model one can make agreement with quantum mechanics as close as desired. In Chapter 5 attempt is made to remove one major trouble in relativistic quantum mechanics-space and time being treated differently. Moreover the author takes Prigogine's suggestion that microscopic physics must include some form of time irreversibility as otherwise the prevalent existence of irreversibility in the universe is difficult to explain. Both objectives are accomplished by assuming that real processes do not take place in space and time but are projected there through the interaction with the observer. The mathematical formulation leads to Schrödinger equation but with modifications in the interpretation of time. In the last chapter Selleri puts forward ideas to show that $\psi$ waves are not merely probability waves. They can also be thought of as real waves and can generate observable effects, These are given the name empty waves as they do not carry energy and momentum. According to this view a quantum particle has a complex structure, a localised particle plus an unlocalised real wave. The author reviews various suggestions about experiments which could lead to the detection of empty waves.

To many physicists the subject of discussion in this book may not appear to be of much consequence. As the editor comments "At first glance it might seem that the research done on interpretative problems has no impact on the more practical problems of modern natural science". The search for a picture of reality in accordance with some pre-conceived notion may not appear to be a compulsive problem. But there are other more pragmatic reasons to believe that quantum mechanics does not provide a completely satisfactory theoretical framework. Particularly the need to have a classical world (in the measurement theory) is disturbing. The conflict with relativity discussed in Chapter 5 is another point to refer. For these and similar other problems, it is necessary that the struggle to go 
beyond the conventional quantum theory must continue. It is in this light that the researches presented in this book derive their real significance.

S SENGUPTA

Condensed Motter Physics Research Centre, Jadavpur University, Calcutta-700 032

\author{
Knowledge-Based Systems in Astronomy (Lecture Note in Physics, Vol 329) \\ edited by $A$ Heck and $F$ Murtagh
}

Springer-Verlag : Berlin-Heidelberg-New York-London-Paris-Tokyo-Hong Kong, 1989

iv + 125 pages ; price : DM 55 (Hard cover) ; ISBN 3-540-51044-3

Observation and data analysis in astronomy have now become rather involved undertakings, because of the giant sizes and technological complexity of the telescopes, many of which are on orbiting platforms in space, and the immense amounts of data that are churned out. Every step of the operation, including making a proposal for observing time, refereeing received proposals, allocating time in the most efficient way, analyzing the output from the observing run, archiving the data for future use, and publishing it in a form which is most suitable for scientific use is extremely involved and time consuming. On the other hand, the number of astronomers who want to have access to state-of-the-art instruments is growing rapidly, and in fact most large and expensive instruments these days are intended for use by the entire community of astronomers. But most aspiring observers would be daunted if they had to spend a great deal of time simply learning how to make proposals in the right format, and how to use instruments, rather than getting on with observation and analysis. It is therefore necessary to evolve systems and procedures which would make it possible for the nature astronomer to use a given instrument with maximum efficiency, without having to spend a great deal of time simply learning techniques. Until just a few years ago the observer could rely on manuals and help provided in an informal manner by friends and experts at the concerned observatory from whom the required skills could be picked up in a short time. But given the gigantic nature of the present projects and the number of people who want to be involved in observing, it has become necessary to resort to automated and more rigorous methods. Expert Systems, or Knowledge Based Systems, seem to be the right tool.

Expert systems are computer programs that include (1) a data base of knowledge derived from the knowledge and experience of human experts, (2) information supplied by the user concerning the application desired, and (3) the computational part of the program which applies the rules in the knowledge base to the information supplied by the user in order to draw conclusions. Expert systems go beyond ordinary computer programs in that they provide a much more subtle 
examination of the problem and the alternative solutions available, and an explanation as to why the provided solution is the best one. Most importantly, the concerned programs are devised in such a manner that they allow user interaction through commands which are closer to natural language commands than what is allowed by less sophisticated programs.

The book under review provides an excellent introduction to the application of Expert Systems (ES) to the various tasks in astronomy. It is divided into four parts : The first summarizes the use of ES in telescope and observatory operations, the second to data analysis, and the third to the classification of galaxies and IUE spectra. The fourth part summarizes some important methods which go in making expert systems, and the last and fifth directions for the future. Each part contains a few chapters, each contributed by one or more experts in the field. Most of the chapters are written in an informal and easy to understand style, and are of modest length.

The book does not begin with an introduction to the concept of expert systems. Chapter 2, following the foreward in Chapter 1, gets straightaway to the application of ES to the amazingly complicated task of alloting time on the Hubble Space Telescope, and scheduling the observations so that optimal use is made of an instrument which is so expensive to run and maintain, and is so heavily oversubscribed. The problems involved here and the difficulties in their solution are easily understood by the reader, so the advantages in the application of expert systems become all the more appealing. Even where the reader is ignorant of what an expert system is, the basic facts can be picked up in a cursory reading of this and some of the other chapters. Chapter 3 deals with the general problem of telescope scheduling, without reference to any particular instrument.

Chapters 8, 9 and 10 provide some examples of the application of ES to the important field of classification in astronomy, by considering the classification of sunspots, galaxies and stellar spectra respectively. It is necessary to classify objects like galaxies so that some pattern may be found in the bewildering variety of forms which presents itself at first sight. The classes then point to the dominance of particular phenomena, making it possible to build simple models using the basic principles of astrophysics. The classification of galaxies was first made by Hubble, and later improved by Sandage, de Vaucouleurs and others. These pioneers depended on relatively small samples at low resolution obtained with great effort. Modern instruments however make it possible to o'stain very large samples, at much higher resolution and dynamic range, so that more detailed classification, involving new principles, can now be attempted. Eut the profusion of data make the task impossible for a human being, and ES have to relied upon. Chapter 9 described some preliminary steps towards automated galaxy classification, while Chapter 10 does the same for spectra obtained using the IUE satellite. 
Chapters 4,5 and 6 discuss analysis, considering in turn surveys of large regions of the sky to map the large scale distribution of matter, tracking the 3-dimensional movement of cosmic rays and finding periodicities in the celestial sources of gamma radiation. Chapter 11 is a formal exposition on the methods to be used whenever inexactness and vagueness are inherent properties of the data, while Chapter 12 describes the use of ES in the automatic interpretation of images. Chapter 13 provides an introduction to neural networks, while possibilities for the future are summarized in Chapter 14, which is the last. This is followed by a glossary and list of acronyms, which are very useful to the beginner.

The chapters in the book are all well-written, though of varying levels of difficulty. The rook is self-contained, in the sense that reading it one can get some flavour of the application of the methods of Expert Systems to astronomy, without having prior knowledge about Expert Systems themselves. Awareness of these techniques can prove to be very beneficial to the modern astronomer. The book is strongly recommended for purchase by libraries which specialize in astronomy and astrophysics, but it may not prove to be very useful in a personal collection.

Ajit Kembahivi Inter-University Centre for Astronomy and Astrophysics, Post Bog 4, Ganeshkhind. Pune-4II 007

\author{
Selected Papers Vol 4: Plasma Physics, Hydrodynamic and Hydro- \\ magnetic Stability and Applications of the Tensor-Virial Theorem. \\ by S Chandrasekhar \\ The University of Chicago Press : Chicago, USA ; 1989 \\ 585 pages ; price : US $\$ 34.50$ (Paper); ISBN 0-226-10097-9
}

Professor Subrahmanyan Chandrasekhar was the first purely theoretical astronomer to be awarded the Nobel Prize in 1983. He has contributed to almost every branch of theoretical astrophysics and his later years are occupied with his investigations in the field of general relativity. He concentrated on a single branch of astrophysics for a few years and produced a series of papers which bear testimony to his remarkable mathematical skill and intuition. After a systematic investigation of the field he published a comprehensive monograch containing the fruits of his labour.' He did this several times.

The present book is volume 4 of a series of volumes of selected papers by him. It contäins a collection of thirtyfive papers published during the period 1954-71. His contributions during the period were published in two monographs viz. "Hydrodynamic and Hydromagnetic Stability" publishe'd in 1961 and "Ellipsoidal Figures of Equilibrium" published in 1969. The papers in the present book were 
selected by N R Lebovitz on the basis of the following criteria : "First, whether the papers have not been included in any of my published books, and second, whether the papers include matters not treated in sufficient detail elsewhere and the possible historical interest they may have". The papers have been divided into three chapters: (1) Plasma Physics, (2) Hydrodynamic and Hydromagnetic Stability and (3) Tensor Virial Theorem and its Applications.

Part I contains four papers published during the period when Prof Chandrasekhar was engaged in investigations of hydrodynamic and hydromagnetic stability but these are not exactly in the direct line of development. Part II contains eight papers not covered in sufficient detail in the monograph and includes one which was published after its appearance. Part III contains twenty three papers on the ellipsoids of McLaurin, Jacobi, Dedekind and Riemann and their stability. Chandrasekhar brought these Riemann ellipsoids, as they are now called, from relative obscurity to the limelight and investigated their properties. It is now frequently referred to in the literature. Later, he included general relativistic corrections in his calculations.

The historian of science will surely welcome the appearance of these volumes. Professor Chandrasekhar has become a legend in his lifetime. Whenever a young scientist takes up research in some branch of astrophysics, he is often asked by his colleagues to find out what contributions Professor Chandrasekhar made to this branch, so versatile has been his interests. A young astrophysicist looks at the huge and diverse material collected in these volumes with awe and despairs of ever becoming a scientist of this class.

$S$ BANERJI

Department of Physics, The University of Burdwan, Golapbogh, Burdwan-7/3104

Far from Equilibrium Phase Transitions (Lecture Notes in Physics, Vol 319)

(Proceedings of the $X$-th Sitges Conference on Statistical Mechanics, Sitges, Barcelona, Spain, June 6-10, 1988)

edited by $L$ Garrido

Springer-Verlag : Berlin-Heidelberg-New York-London-Paris-Tokyo, 1988

viii + 340 pages; price : DM 65 (Hard cover) : ISBN 3-540-50643-8

The far from equilibrium phase transition is one of the most active area of research today. A variety of interesting phenomena in the diverse field of optics, fluid mechanics, chemistry, biology etc. offer good opportunity to unify themselves on this common theoretical ground. For example, thə laser threshold instability, the transition to chaos in the laser itself, instabilities in passive systems as in optical instability are prototype for such far-from-equilibrium phase transitions in 
optics. Similarly, in autocatalytic chemical reactions or in biological systems with reproduction processes, one can find interesting situations which bear close analogies with second order phase transition. Also there are classic age-old problem of Rayleigh-Benard convection, Couette-Taylor flow in hydrodynamics. A common ingradient for all these problems is the nonlinearity. Nonlinearity together with suitable boundary effects or with dispersion make these situations interesting from the point of view of pattern formation, structure and self-organization. Since second order phase transition is derivable from Ginzburg-Landau theory, bifurcation theory also comes into the picture. The present volume of conference proceedings of Springer-Verlag provides an excellent set of lectures devoted to these problems.

The volume starts with a review on the current problems in the theory of envelope solutions. Four articles deal with nonlinear optical systems relating optical instability, fluctuation in the transient dynamics, phase and frequency dynamics in laser instabilities, spontaneous symmetry-breaking and spatial structures. Phase transitions and fluctuations have been treated in reactiondiffusion systems, in binary mixtures, in the interfacial problems etc. A number of articles treat some theoretical methods on ho' $\mathrm{N}$ in general patterns are formed, on exact solvability of some multistate Fokker-Planck models, on statistical properties of the retarded equations, on time-dependent bifurcations and on technique for the solution of Fokker-Planck equation by matrix continued fraction.

The most of the articles are excellent from the point of view of presentation, many of which would be appreciated even by very young research workers. However, some classification of the articles in several sections would have been more helpful for a general reader. This book would certainly be a welcome addition to any library of Physical Science.

\author{
D S RAY \\ Department of Physical Chemistry. \\ Indian Association for the Cultivation of Science, \\ Jadovpur, Calcutta-700 032
}

\title{
The Physical Basis of the Direction of Time
}

by H D Zeh

Springer-Verlag : Berlin-Heidelberg-New York-London-Paris-Tokyo-Hong Kong, 1989 viii + 166 pages, 20 figures ; price : DM 56 (Soft cover) ; ISBN 3-540-50930-5

This book is a 'revised and extended' English version of the German publication Die Physik der Zeitrechtung (Vol 200 of the Springer Lecture notes in Physics) which arose from a course of lectures at the University of Heidelberg. We are not told about the type of audience to which the lectures were addressed but the 
author expresses his intention to point out the essential physical ideas rather than to describe the technical or mathematical aspects and he aims at one who is interested in an overview of the whole problem. However, when one just glances over the topic headings as given in the contents, one finds that not only the cases of time arrow in electromagnetism, thermodynamics, quantum mechanics and expanding universe models are considered in detail but more sophisticated and highly technical developments like Everett's ideas and quantum gravity and cosmology are also brought in. Indeed the reviewer doubts whether without knowing technicalities one can have a flavour of these developments.

The concept of time is perhaps the most difficult one to analyse and the average reader is sure to be bewildered by the quotation from Einstein in Epilogue. "For us believing physicists, the division into past, present and future has merely the meaning of an albeit obstinate illusion".

There is a rather select group of people, (to which the present reviewer does not belong) who can go deep into the profound problem which forms the subject matter of the book. They will surely enjoy sharing the ideas with a fellow thinker.

A K RAYChAUDHURI

Department of Physics, Presidency College, Calcutta-700 073 